\title{
Research on Optical Fiber Flow Test Method With Non-Intrusion
}

\author{
Ying SHANG ${ }^{1,2^{*}}$, Xiaohui $\mathrm{LIU}^{1,2}$, Chang $\mathrm{WANG}^{1,2}$, and Wenan $\mathrm{ZHAO}^{1,2}$ \\ ${ }^{1}$ Laser Research Institute of Shandong Academy of Sciences, Jinan, 250014, China \\ ${ }^{2}$ Key Laboratory of Optical Fiber Sensoring Technology of Shandong Province, Jinan, 250014, China \\ *Corresponding author: Ying SHANGＥ-mail: sy81012607@163.com
}

\begin{abstract}
In the field of oil well logging, real-time monitoring of the fluid flow parameter provides a scientific basis for oil and gas optimization exploration and increase in reservoir recovery, so a non-intrusive flow test method based on turbulent vibration was proposed. The specific length of the sensor fiber wound tightly around the outer wall of the pipe was connected with the optical fiber gratings at both ends, and the sensor fiber and the optical fiber gratings composed the flow sensing unit. The dynamic pressure was generated by the turbulence when fluid flows through the pipe, and the dynamic pressure resulted in the light phase shift of the sensor fiber. The phase information was demodulated by the fiber optic interferometer technology, time division multiplexing technology, and phase generated carrier modulation and demodulation techniques. The quadratic curve relationship between the phase change and flow rate was found by experimental data analysis, and the experiment confirmed the feasibility of the optical fiber flow test method with non-intrusion and achieved the real-time monitoring of the fluid flow.
\end{abstract}

Keywords: Oil, flow, turbulent vibration, the fiber optic interferometer, phase generated carrier

Citation: Ying SHANG, Xiaohui LIU, Chang WANG, and Wenan ZHAO, "Research on Optical Fiber Flow Test Method With Non-Intrusion," Photonic Sensors, 2014, 4(2): 132-136.

\section{Introduction}

In the oil field development and production, pressure, temperature, and flow are important parameters of the well. Real-time monitoring of those important parameters provides a scientific basis for oil and gas optimization exploration and an increase in reservoir recovery. In the oil industry, because of the complex characteristic of the fluid composition and various flow states and bad working condition, the conventional electronic sensors can not work in the underground environment such as high temperature, high pressure, corrosion, and electrical interference [1-5].
A new kind of the optical fiber monitoring system of fluid flow using flow-induced pipe vibration was proposed. By optimizing the design flow sensor, a turbulent flow device was placed in front of the sensor unit to enhance the turbulence intensity, then a flow measurement has completed, and the system has extensive application prospects in the oil field development and production because of high sensitivity, high accuracy, high stability.

\section{Measure principle of the system}

\subsection{Principle of optical phase modulation}

The optical phase depends on three

Received: 29 December 2013 / Revised version: 18 January 2014

(C) The Author(s) 2014. This article is published with open access at Springerlink.com DOI: $10.1007 / \mathrm{s} 13320-014-0177-0$

Article type: Regular 
characteristics of the optical waveguide when light transmits in the fiber: the total physical length, refractive index, and lateral dimensions of the optical waveguide. The waveguide is supposed to be monochromatic when light transmits in the optical fiber, and its wavelength in the air is $\lambda_{0}$.

Optical waveguide refractive index distribution is supposed to be constant with the external changes, the disturbance is applied to the fiber, and the optical phase sensitivity corresponding to the disturbance can be estimated. After transmission through the $L$-meter length of the fiber, the phase delay of the outgoing light [6] is

$$
\phi=2 \pi n L v / c
$$

where $n$ is the refractive index of the fiber core, $c$ is the speed of light in vacuum, and $v$ is the optical frequency. Obviously, $L, v$, and $n$ will result in changes in the output optical phase, which can be seen from (1):

$$
\Delta \phi=\frac{2 \pi n L v}{c}\left(\frac{\Delta n}{n}+\frac{\Delta L}{L}+\frac{\Delta v}{v}\right) .
$$

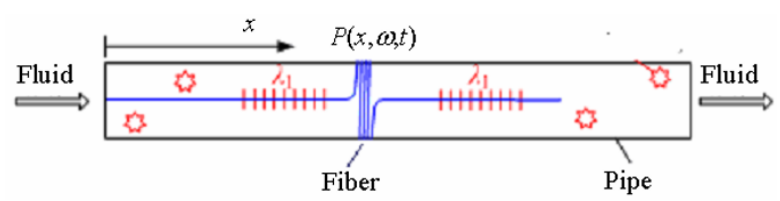

Fig. 1 Principle diagram of the flow sensor.

Phase modulation is usually caused by physical factors such as stress-strain and temperature effects. The phase change caused by the temperature effect mainly relates to the impact of the external environment on the system, so the temperature effect needs to be fully considered in the system. However, the process of the phase change caused by the temperature effect is slow, so signal processing can be used to solve the phase change caused by the temperature effect. The phase change caused by stress-strain is mainly in the new optical fiber monitoring system of fluid flow in this paper. The optical fiber is wrapped closely around the outside wall of the pipe between pairs of low reflectivity fiber Bragg gratings as shown in Fig. 1. When the pressure field $P(x, w, t)$ changes in the pipe, the change in fiber strain causes the shift of the interferometer phase variation. The pressure signal $P(x, w, t)$ at the coordinate $x$ is obtained after demodulation.

\subsection{Modulation and demodulation techniques of phase generated carrier}

The interferometer output signal $I$ can be expressed as

$$
I=A+B \cos \varphi(t)
$$

where $A$ is the average optical power of the interferometer output signal, $B=\kappa A, \kappa \leq 1, \kappa$ is the interference fringe visibility, and $\cos \varphi(t)$ is the phase variation of the interferometer.

Let $\varphi(t)=C \cos \left[\omega_{0} t+\phi(t)\right]$, then (3) can be written as $[7,8]$

$$
I=A+B \cos \left[C \cos \omega_{0} t+\phi(t)\right]
$$

where $C \cos \omega_{0} t$ is the phase generated carrier, $\phi(t)=D \cos \omega_{s} t+\psi(t), \quad D \cos \omega_{s} t \quad$ is the phase variation caused by the tested signal, and $\psi(t)$ is the slow variation of the initial phase caused by environmental disturbances.

With the Bessel function expansion, (4) can be expressed as

$$
\begin{aligned}
I= & A+B\left\{\left[J_{0}(C)+2 \sum_{k=1}(-1)^{k} J_{2 k}(C) \cos 2 k \omega_{c} t\right] \cos \varphi_{s}(t)\right. \\
& \left.-2\left[\sum_{k=0}^{\infty}(-1)^{k} J_{2 k+1}(C) \cos (2 k+1) \omega_{c} t\right] \sin \varphi_{s}(t)\right\} .
\end{aligned}
$$

The transfer process of the signal $I$ is shown in Fig. 2, so the final output of the system which contains the tested signal $D \cos \omega_{s} t$ is

$$
S(\omega, t)=B^{2} G H J_{1}(C) J_{2}(C) D \cos \omega_{s} t .
$$

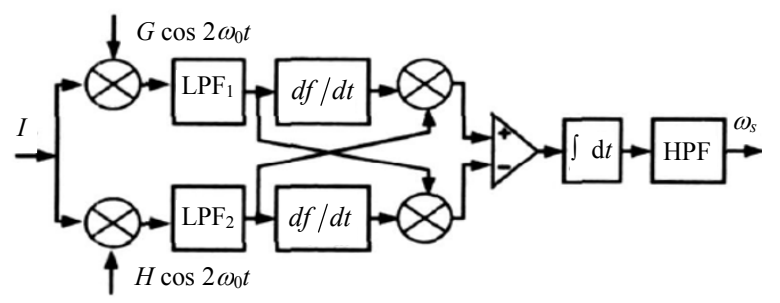

Fig. 2 Block diagram of the phase generated carrier (PGC).

\subsection{Pressure testing principle}

As the molecules of the fluid approach the pipe 
wall, they have kinetic energy. This kinetic energy must be converted to another form of energy as the molecules reach the pipe wall. According to the first law of thermodynamics, some of the kinetic energy is converted to heat as the turbulent eddies dissipate, but most is converted into potential energy in the form of pressure.

According to Prashu's research on turbulent flow, the instantaneous fluctuations pressure $p$ is proportional to the flow rate as shown by (7) [9]:

$$
p \propto \bar{u} \bar{v} .
$$

In (3), $\bar{u}$ is the velocity in the direction of the primary pipe axis, and $\bar{v}$ is the velocity perpendicular to the pipe axis.

The fluid filled piping system can be depicted as a one-dimensional model of a beam, so we can get the relation as shown by (8):

$$
\frac{d^{2} M}{d x^{2}}=\frac{d V}{d x}=p^{\prime}(x)
$$

where $d^{2} M / d x^{2}$ is the rate of the change in the moment along a beam, $d V / d x$ is the rate of the change in shear, and $p^{\prime}(x)$ is the fluctuations pressure per unit length.

Combined with the general formula of engineering mechanics, (9) can be deduced:

$$
E I=\frac{d^{4} y}{d x^{4}}=p^{\prime}(x) .
$$

In order to relate the pressure fluctuations to the pipe acceleration $d^{2} y / d t^{2}$, consider the differential equation of motion for transverse vibration of a beam in (10):

$$
\frac{\partial^{2} y}{\partial t^{2}}=-\frac{g}{A \gamma} E I \frac{\partial^{4} y}{\partial x^{4}}
$$

It is well known in (11) that the acceleration of the pipe is proportional to the pressure fluctuation in the fluid.

$$
p^{\prime}(x) \propto \frac{\partial^{2} y}{\partial t^{2}} .
$$

According to the derivaton of the turbulence intensity in [10], we can get

$$
\sqrt{\frac{1}{N-1} \sum_{i=1}^{N}\left[u_{i}(t)-\bar{u}\right]^{2}} \propto \bar{u} .
$$

Since the flow fluctuations are proportional to the pressure fluctuations and the pressure fluctuations are proportional to the pipe vibrations, it follows that the standard deviation of the pipe vibrations is proportional to the average flow rate.

\section{Experiment}

\subsection{Design of new optical fiber monitoring system}

As shown in Fig. 3, the continuous optical signal is modulated into the pulse sequence whose frequency is $100 \mathrm{kHz}$, and the duty cycle is 0.1 . The system includes a two-beam interferometer which comprises an optics circuit for generating a series of discrete light pulses that are directed at sensors positioned between pairs of fiber Bragg gratings. The sinusoidal modulation signal whose frequency is $40 \mathrm{kHz}$ is modulated on a beam of the MachZender interferometer, while the other beam is made up of the $105-\mathrm{m}$ fiber. The optical fiber delay produces two light pulses, a group of light pulses composed of the three pulse sequences can be obtained. The pulse 2 contains the information of the sensor fiber 1 , and the pulse 3 contains the information of the sensor fiber 2. The interference signal is processed through the phase generated carrier and related pressure testing algorithms, finally, the size of fluid flow is accurately determined with non-intrusion.

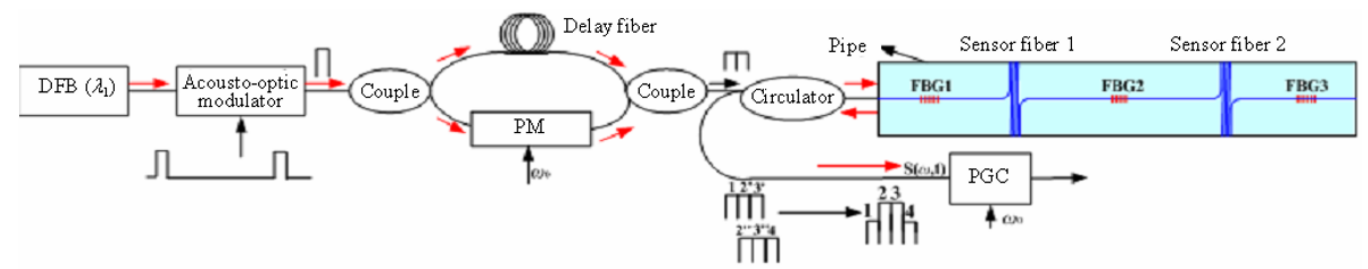

Fig. 3 Schematic diagram of the optical fiber grating interferometer. 


\subsection{Experimental results}

The experimental system was built as shown in Fig. 4. The pump drew water in the circulation, the flow was changed by adjusting the valve in the experiment, the electronic flow meter calibrated the real flow value, then the relationship between the flow value and the corresponding light intensity was monitored by the optical fiber fluid flow monitoring system.

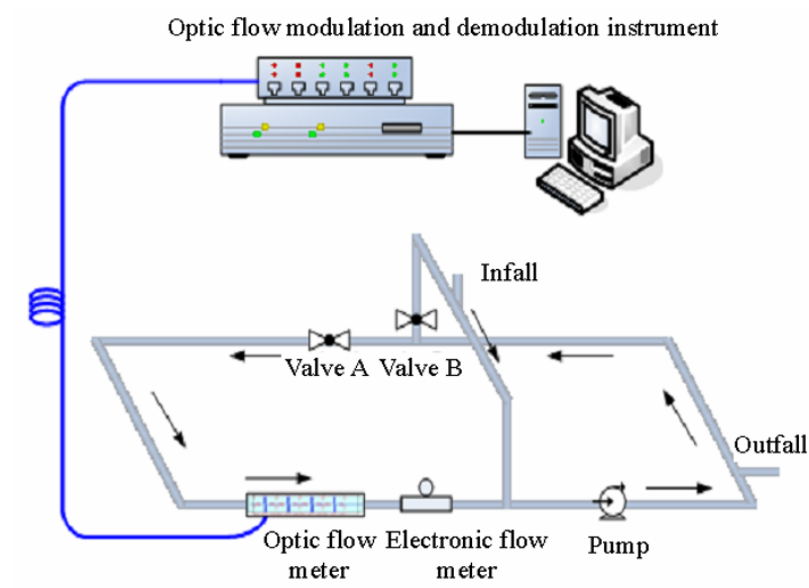

Fig. 4 Flow experimental system diagram.

In this experiment, the demodulated signal was analyzed to determine the vibration frequency characteristics of a pipe in the frequency domain, then the flow signal was solved in the corresponding frequency domain. The flow experimental data are shown in Table 1, Table2, and Table3. As shown in Fig. 5(a), the signal frequency range is between $1 \mathrm{kHz}$ and $10 \mathrm{kHz}$, and its amplitude becomes larger because of the environment low-frequency noise, so

Table 1 Flow experimental data in the frequency range of $1 \mathrm{kHz}-10 \mathrm{kHz}$.

\begin{tabular}{cccc}
\hline Flow $\left(\mathrm{m}^{3} / \mathrm{h}\right)$ & light intensity $(\mathrm{V})$ & Flow $\left(\mathrm{m}^{3} / \mathrm{h}\right)$ & light intensity $(\mathrm{V})$ \\
\hline 5.7 & 0.053366 & 44.1 & 0.315767 \\
10.8 & 0.1241 & 39.1 & 0.285697 \\
16.8 & 0.159968 & 35.4 & 0.268336 \\
19.8 & 0.1448 & 30.9 & 0.235911 \\
25.8 & 0.1845 & 25.8 & 0.190294 \\
29.8 & 0.22649 & 26.1 & 0.18291 \\
34.9 & 0.264839 & 18.8 & 0.150126 \\
40.6 & 0.297049 & 15.9 & 0.155238 \\
44.4 & 0.311624 & 12.6 & 0.1371 \\
46.5 & 0.319 & & \\
\hline
\end{tabular}

Table 2 Flow experimental data in the frequency range of $10 \mathrm{kHz}-20 \mathrm{kHz}$

\begin{tabular}{cccc}
\hline Flow $\left(\mathrm{m}^{3} / \mathrm{h}\right)$ & light intensity $(\mathrm{V})$ & Flow $\left(\mathrm{m}^{3} / \mathrm{h}\right)$ & Light intensity $(\mathrm{V})$ \\
\hline 5.7 & 0.005026 & 44.1 & 0.02836 \\
10.8 & 0.007256 & 39.1 & 0.026703 \\
16.8 & 0.011102 & 35.4 & 0.02475 \\
19.8 & 0.013111 & 30.9 & 0.022915 \\
25.8 & 0.017417 & 25.8 & 0.018267 \\
29.8 & 0.020569 & 26.1 & 0.016528 \\
34.9 & 0.025079 & 18.8 & 0.012537 \\
40.6 & 0.027462 & 15.9 & 0.010512 \\
44.4 & 0.028674 & 12.6 & 0.008928 \\
46.5 & 0.031389 & & \\
\hline
\end{tabular}

Table 3 Flow experimental data in the frequency range of $10 \mathrm{kHz}-12 \mathrm{kHz}$.

\begin{tabular}{cccc}
\hline Flow $\left(\mathrm{m}^{3} / \mathrm{h}\right)$ & light intensity $(\mathrm{V})$ & Flow $\left(\mathrm{m}^{3} / \mathrm{h}\right)$ & light intensity $(\mathrm{V})$ \\
\hline 5.7 & 0.004855 & 44.1 & 0.030154 \\
10.8 & 0.006602 & 39.1 & 0.025826 \\
16.8 & 0.010064 & 35.4 & 0.024343 \\
19.8 & 0.012361 & 30.9 & 0.021472 \\
25.8 & 0.01713 & 25.8 & 0.017572 \\
29.8 & 0.020098 & 26.1 & 0.016577 \\
34.9 & 0.024733 & 18.8 & 0.011459 \\
40.6 & 0.026956 & 15.9 & 0.009985 \\
44.4 & 0.030796 & 12.6 & 0.007777 \\
46.5 & 0.031421 & & \\
\hline
\end{tabular}

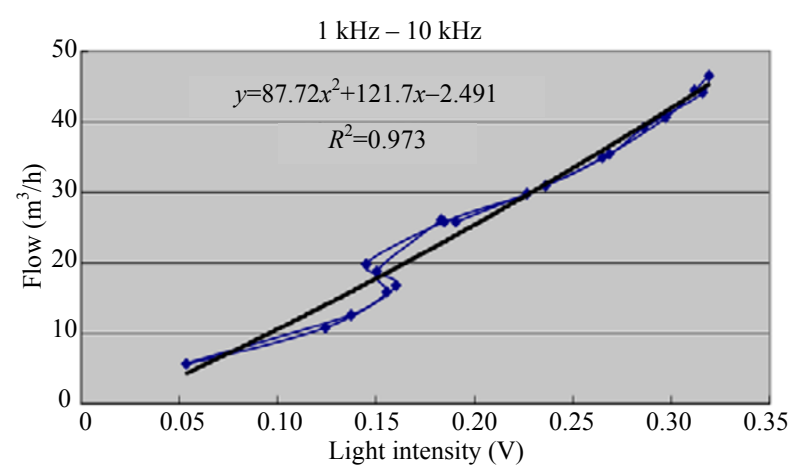

(a)

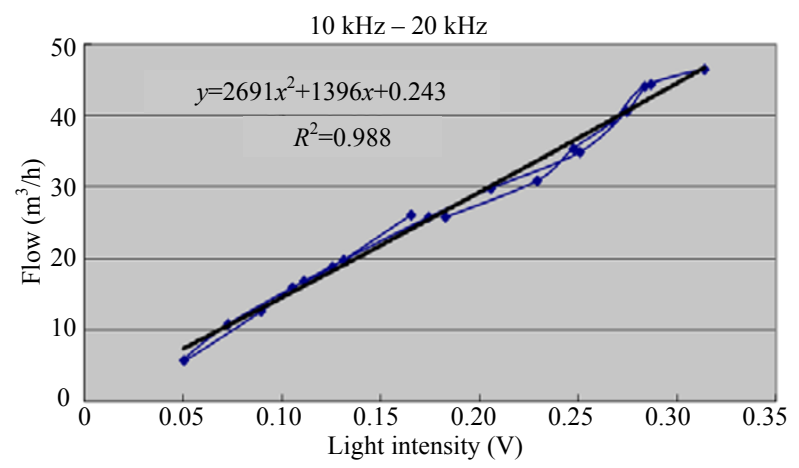

(b)

Fig. 5 Relationship diagram between flow and light intensity in different frequency range. 
the curve fitting of flow is not very good. In Fig. 5 (b), the signal frequency range is between $10 \mathrm{kHz}$ and $20 \mathrm{kHz}$, so the curve fitting of flow is better than that in Fig.5(a), In summary, the vibration frequency range of a pipe is between $10 \mathrm{kHz}$ and $12 \mathrm{kHz}$, In this frequency range, the quadratic curve relationship between the flow and light intensity is shown in Fig. 6.

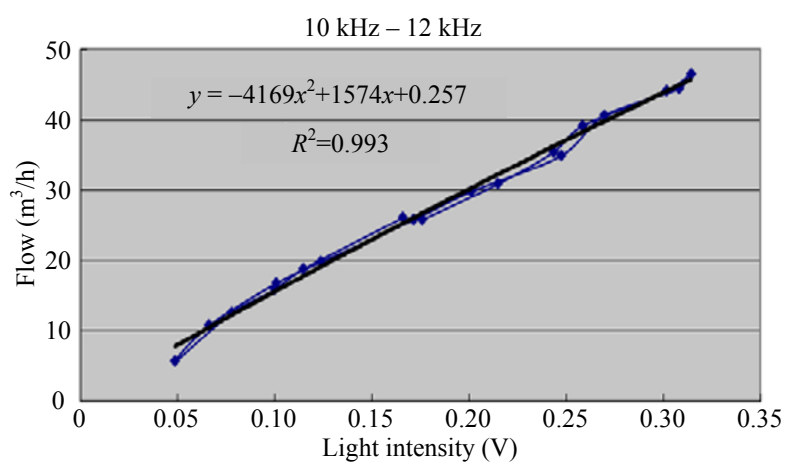

Fig. 6 Relationship diagram between the flow and light intensity in the frequency range of $10 \mathrm{kHz}-12 \mathrm{kHz}$.

\section{Conclusions}

This paper describes a new optical fiber monitoring system of fluid flow used in the oil well logging field in detail, and the size of fluid flow was accurately measured with non-intrusion, and the information of fluid flow provides the scientific basis for the optimization of the oil and gas exploration program and an increase in reservoir recovery.

\section{Acknowledgment}

This work was financially supported by the Doctor Fund of Shandong Province: "the fiber optic flow meter downhole and oil interferometer demodulation techniques" (2009BSC01028) and sustentation fund of Sinopec Group "fiber four-parameter monitoring technology of oil wells".

Open Access This article is distributed under the terms of the Creative Commons Attribution License which permits any use, distribution, and reproduction in any medium, provided the original author(s) and source are credited.

\section{References}

[1] M. Liu, F. Zhou, and Z. Du, "Application of optic fiber sensors in petrol well logging," Optics \& Optical Electronic Technology, 2008, 6(3): 18-21.

[2] Q. Zhang, X. Qiao, and H. Fu, "Advances in fiber-optic flowmeters," Study on Optical Communications, 2007, 141(3): 58-61.

[3] W. Wang and X. Liu, "Effects of azimuth of fiber optic principal axes on fiber optic Current sensor," Chinese Journal Lasers, 2013, 40(1): 0114003.

[4] Z. Zhao, P. Wu, and C. Sui, "A novel microfiber sensor based on white light interferometer," Chinese Journal Lasers, 2010, 37(8): 2001-2004.

[5] B. Qi, F. Pang and W. Cao. "Cladding-mode resonance of specialty fiber sensing system based on intensity detection method," Chinese Journal Lasers, 2011, 38(5): 0505007.

[6] S. Peng, "Study on fiber bragg grating stress sensing," Journal of Hunan Institute of Science and Technolog (Natural Sciences), 2007, 20(2): 36-38.

[7] A, Dandridge, A. B. Tvcten, and T. G. Giallorenzi, "Homodyne demodulation sehemes for fiber optic sensor susing phase generated carrier," IEEE Journal Quantum Electronics , 1982, 18(10): 1647-1651.

[8] L. Wang, J. He, and F. Li, "Ultra low frequency phase generated carrier demodulation technique for fiber sensors," Chinese Journal Lasers, 2011, 38(4): 0405001.

[9] M. T. Pittard, R. P. Evans, R. D. Maynes, and J. D. Blotter, "Experimental and numerical investigation of turbulent flow induced pipe vibration infully developedflow," Review of Scientific Instruments, 2004, 75(7): 2393-2041.

[10] R. P. Evans, J. D. Blotter, and A. G. Stephens, "Flow rate measurements using flow induced pipe vibration," Journal of Fluids Engineering, 2004, 126(2): 280-285. 